Recepción: 14 / 06 / 2018

Aceptación: 22 / 08 / 2018

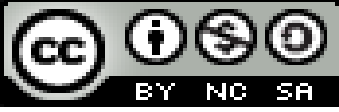

Publicación: 05 / 10 / 2018

\title{
Los proyectos de investigación social en el contexto de la extensión Universitaria
}

\section{Social research projects in the context of University extension}

\section{Projetos de pesquisa social no contexto da extensão Universitária}

\author{
Alba D. Alay-Giler ${ }^{\mathrm{I}}$ \\ aalay@utm.edu.ec \\ Hipatia A. Meza-Intriago ${ }^{\text {II }}$ \\ hmeza@utm.edu.ec \\ Zoila I. Franco-López III \\ zoifrancolopez@gmail.com \\ Mayra A. Tejena-Macías IV \\ mayratejena@gmail.com
}

Correspondencia: aalay@utm.edu.ec

\footnotetext{
I Magíster en Gerencia de Proyectos Educativos y Sociales, Licenciada en Ciencias de la Educación Espec. Física y Matemáticas, Profesor de Segunda Enseñanza Especialidad Física y Matemáticas, Docente de la Universidad Técnica de Manabí, Portoviejo, Ecuador.

${ }^{\text {II }}$ Doctor en Educación, Magíster en Educación Parvularia, Licenciada en Ciencias de la Educación Esp. Química y Biología, Profesora de Segunda Enseñanza, Docente de la Universidad Técnica de Manabí, Portoviejo, Ecuador.

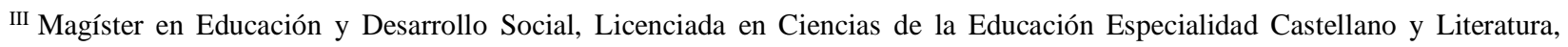
Profesor de Segunda Enseñanza Especialidad Castellano y Literatura, Docente de la Universidad Técnica de Manabí, Portoviejo, Ecuador.

IV Magíster en Tecnología E Innovación Educativa, Ingeniera en Sistemas, Docente de la Universidad Laica Eloy Alfaro de Manabí, Manta, Ecuador.
} 


\section{Resumen}

La sociedad exige de las universidades repuestas a sus necesidades sociales, y ello solo es posible a través de los procesos de investigación que en ella se cumplen. Esta premisa, permitió estudiar las características de relación entre los proyectos de investigación universitaria y su entorno, específicamente en el contexto de la extensión universitaria. Con base a lo expuesto, se desarrolló un estudio de tipo documental, con un enfoque hermenéutico. El corpus de estudio lo constituyeron los proyectos de investigación social, en el contexto de la extensión universitaria. La metodología a utilizar fue de tipo cualitativa y la técnica documental. La información se obtuvo a través de una revisión bibliográfica-documental de los aspectos académicos que constituyen los proyectos de investigación social en la Universidad. El análisis de la información, permitió exponer de forma práctica la elaboración de un proyecto de investigación social.

Palabras clave: proyectos; investigación social; aspectos académicos; universidad.

\section{Abstract}

Society demands from universities that respond to their social needs, and this is only possible through the research processes that are carried out in it. This premise allowed us to study the characteristics of the relationship between university research projects and their environment, specifically in the context of university extension. Based on the above, a documentary study was developed, with a hermeneutical approach. The corpus of study was constituted by social research projects, in the context of university extension. The methodology to be used was qualitative and the documentary technique. The information was obtained through a literaturedocumentary review of the academic aspects that constitute the projects of social research in the University. The analysis of the information allowed to expose in a practical way the elaboration of a social research project.

Key words: projects; social investigation; academic aspects; college.

\section{Resumo}

A sociedade exige das universidades que respondam às suas necessidades sociais, e isso só é possível através dos processos de pesquisa que são realizados nela. Essa premissa nos permitiu 
Alba D. Alay Giler, Hipatia A. Meza Intriago, Zoila I. Franco López, Mayra A. Tejena Macías estudar as características da relação entre os projetos de pesquisa universitária e seu ambiente, 
especificamente no contexto da extensão universitária. Com base no exposto, foi desenvolvido um estudo documental, com abordagem hermenêutica. $\mathrm{O}$ corpus de estudo foi constituído por projetos de pesquisa social, no contexto da extensão universitária. A metodologia a ser utilizada foi qualitativa e a técnica documental. As informações foram obtidas através de uma revisão bibliográfica-documental dos aspectos acadêmicos que constituem os projetos de pesquisa social na Universidade. A análise das informações permitiu expor de maneira prática a elaboração de um projeto de pesquisa social.

Palavras chave: projetos; pesquisa social; aspectos acadêmicos; universidade.

\section{Introducción}

El proyecto de investigación explica Hurtado (2010), se constituyen en procedimientos científicos que permiten obtener información y hasta formular hipótesis sobre un determinado fenómeno social o científico. En el marco de los proyectos de extensión universitaria representan un tipo particular de proyecto, dado que están fundamentado por dos elementos: la voluntad de contribuir a una determinada transformación social y por estar concebidos y desarrollados en torno a la idea de integralidad funcional delos componentes de las funciones universitarias de extensión, investigación y docencia. Ello debido a la noción de extensión universitaria, que según Balestrini (2008), refiere al conjunto de actividades de colaboración de actores universitarios con otros actores que, en procesos interactivos donde cada actor aporta sus saberes y todos aprenden, contribuyen a la creación cultural y al uso socialmente valioso del conocimiento, con prioridad a los sectores más postergados. Siendo así, se propuso en este artículo estudiar las características de relación entre los proyectos de investigación universitaria y su entorno, específicamente en el contexto de la extensión universitaria, para ello se presentan los pasos a seguir en un proyecto de investigación social universitario.

\section{Desarrollo}

Pasos a seguir en un proyecto de investigación social

El proyecto de investigación, constituye el plan de trabajo o de actividades, donde se prevé la duración del proceso investigativo. De esta manera, el investigador trabaja de acuerdo a un 
cronograma que debe respetar y cumplir. El mismo debe componerse de una serie de pasos, entre estos; la selección del tema e identificación de un problema. Para formular el proyecto, el investigador social, ha de realizar un borrador con las ideas básicas de la investigaciones para poder seguir los pasos y realizar las preguntas pertinentes en cada uno de ellos, en cuanto a la redacción del proyecto con características científicas, se debe presentar un proceso compuesto por una serie de etapas secuenciadas, enlazadas unas con otras, las que no pueden ser omitidas o alteradas en su orden; de no ser así, la resultante de la investigación no sería válida ni comparable.

Estas etapas, se combina de forma secuencial tal como lo plantea Méndez (2004), se debe realizar el planteamiento del problema, con la formulación del fenómeno que se investigará. En la siguiente etapa, es necesario establecer los objetivos, es decir, estipular qué se pretende conocer con la investigación. Posteriormente, se plantean la o las hipótesis del trabajo, la cual se formula como la teoría a comprobarse durante el proyecto de investigación. Así como la justificación y delimitación del estudio.

Seguidamente, se incluye el marco teórico y de referencia del proyecto de investigación, en la cual se supone la inclusión de citas y ejemplos de otras investigaciones. Estas, en los proyectos de investigación social funcionan como una referencia general del tema a tratar, con datos que permiten entenderlo con mayor facilidad.

Una vez llegado el momento del trabajo de campo, se inicia la obtención de los datos cuantitativos y cualitativos del tema. Podría decirse que éste es el momento principal de la investigación, donde se realizan los experimentos, las pruebas o las entrevistas necesarias para la obtención de la información. Finalmente llega el turno de la estructuración del trabajo y su redacción final. De este modo, la investigación puede ser presentada en diversos capítulos, con la introducción y una conclusión, entre otros segmentos. 


\section{Organización y componentes de un proyecto de extensión:}

\section{Construcción del problema de intervención}

A la hora de comenzar un proyecto lo relevante es la definición clara y precisa del problema de intervención, plantea Ibáñez (2001), que este aspecto será el orientador para la formulación de los restantes componentes. El problema de intervención, expone Ameigeiras (2006), es la identificación de una situación social particular y concreta, con el objetivo de contribuir a su transformación o de generar las condiciones para ello. Un proyecto puede partir de un tema general y abstracto, de un problema concreto o una necesidad detectada. Independientemente de su origen, el problema debe quedar claramente definido. Por otro lado, el problema no es neutro, pues no existe con independencia de quien interviene.

De allí que, un problema de intervención es tal porque quienes intervienen así lo han comprendido y definido. Este aspecto condicionará las acciones que se llevarán adelante con miras a modificar esa realidad sobre la que se intervendrá. Tal apreciación tiene como correlato una 'ruptura', lo que en términos de Hernández y col (2014), queda expresado como la formulación de Proyectos de Extensión Universitaria, en la que la realidad de la vida cotidiana se da por establecida como realidad, en tanto que no requiere verificaciones adicionales sobre su sola presencia y más allá de ella.

\section{Identificación del problema social}

El proceso de construcción del problema de intervención implica diferenciar el problema social del problema de intervención propiamente dicho. No es posible pensar en un problema de intervención si no lo contextualizamos. En otras palabras, el problema de intervención se inscribe en un problema de mayor envergadura. Este movimiento nos coloca en el plano del problema social. El problema social, explica Tommasino (2009), contextualiza al problema de intervención, permite comprender y aprehender la complejidad en la que se subsume. Mientras el problema social es amplio, el problema de intervención es acotado al ámbito de las acciones concretas. Es el movimiento entre el problema social y el problema de intervención el que permite reflexionar y concluir sobre el alcance d de las acciones, para orientarlas de acuerdo a los objetivos del proyecto. Una forma práctica de delimitar el problema de intervención -diferenciándolo del 
problema social- es identificar las causas o factores que generan dicho problema, el ¿por qué se produce? y los efectos o consecuencias que produce ¿qué efectos produce?

\section{Estrategia de intervención y diseño metodológico}

La propuesta metodológica de un proyecto, refiere Hernández y col (2014), a cómo llevarlo adelante. Implica articular los componentes y aspectos que intervienen en el proyecto de manera que se traduzcan de forma coherente en las acciones concretas durante el transcurso de su ejecución. El diseño de un proyecto no es solo la formulación de un plan de acción, es también la propuesta de inicio de un proceso del que no puede anticiparse totalmente su alcance. En el caso de los proyectos de extensión este aspecto es especialmente relevante en tanto es una práctica reflexiva y dialógica. Esto no implica que sea irrelevante planificar o trazar planes de acción, sino que estas propuestas deben pensarse en términos de generar las condiciones propicias para la construcción de un proceso colectivo. En una estrategia de intervención existen definiciones orientadoras de la acción que refieren, entre otras, a las siguientes cuestiones:

¿Cómo se realizará la entrada al campo de intervención?

¿Cómo afectará al diseño del proyecto los ajustes que se deban realizar en el proceso de construcción de la demanda?

¿Qué estrategias se utilizarán para reflexionar sobre el proceso?

¿Cómo y con quiénes se monitoreará y evaluará lo realizado?

¿Cuáles serán las estrategias para la salida del campo una vez finalizado el proyecto?

En la formulación de un proyecto es necesario expresar un diseño metodológico, es decir un plan de acción. Este diseño o plan presenta diferentes niveles de abstracción.

\section{Organización del trabajo}

La organización del trabajo constituye un aspecto sumamente importante en la realización de un proyecto. Dicha organización refiere al equipo de trabajo y a las tareas que se van a desarrollar. No se trata de un aspecto que permanece estático una vez definido -en este sentido debe ser 
entendido como proceso-. Por el contrario, la organización del trabajo es dinámica, porque se modifica y ajusta a la realidad sobre la que estamos interviniendo. Pero incluso esta flexibilidad debe estar prevista de antemano por el equipo de trabajo. Entre los elementos a considerar al momento de organizar el trabajo en un proyecto, son los siguientes: Planificación, Cronograma, Registro, Evaluación y monitoreo y Recursos disponibles y presupuesto.

\section{A manera de conclusión}

En el presente artículo, se evaluaron las teorías que hacían referencias a los proyectos de investigación social, específicamente en lo que concierne a la función de extensión universitaria, haciendo posible presentar un recorrido de acción desde el punto de vista teórico y práctico de un proyecto de investigación, en la que se propone el curso que se debe llevarse en las instituciones universitarias y así distinguir las diferentes etapas que se cumplen, dado su carácter social dentro del marco de la función de extensión universitaria.

\section{Referencias Bibliográficas}

Ameigeiras, A. (2006). “El abordaje etnográfico en la investigación social”. En Vasilachis de Gialdino (coord.). Estrategias de investigación cualitativa. Gedisa, Barcelona

Balestrini, M. (2008) ¿Cómo se elabora el proyecto de investigación? 6ta Edición. México. Editorial McGraw Hill.

Hernández, Fernández y Baptista (2014). Metodología de la Investigación. Tercera Edición. México. Editorial Me Graw Hill.

Hurtado, J. (2010). Metodología de la Investigación. Editorial Quirón. 6ta edición. Colombia.

Méndez, C. (2004). Metodología de la investigación. Colombia. Editorial Mc Graw Hill.

Tmmasino, H. (2009). “Generalización de las prácticas integrales. Los aportes de la extensión para su implementación”. En Revista en Diálogo, Año 1, No 3. Extensión Universitaria, Montevideo.

Retamozo, M. (2009). “Orden social, subjetividad y acción colectiva. Notas para el estudio de los movimientos sociales". En Revista Athenea Digital No 16: 95-124. Universidad Autónoma de Barcelona, España. Recuperado en: http://www.redalyc.org/ pdf/537/53712934010.pdf 